\title{
Emotion Classification through Nonlinear EEG Analysis Using Machine Learning Methods
}

\author{
Morteza Zangeneh Soroush ${ }^{1}$, Keivan Maghooli ${ }^{1 *}$, Seyed Kamaledin Setarehdan², Ali Motie Nasrabadi ${ }^{3}$ \\ 'Department of Biomedical Engineering, Science and Research Branch, Islamic Azad University, Tehran, Iran \\ ${ }^{2}$ Control and Intelligent Processing Centre of Excellence, School of Electrical and Computer Engineering, College of \\ Engineering, University of Tehran, Tehran, Iran \\ ${ }^{3}$ Department of Biomedical Engineering, Faculty of Engineering, Shahed University, Tehran, Iran
}

\begin{abstract}
Background: Emotion recognition, as a subset of affective computing, has received considerable attention in recent years. Emotions are key to human-computer interactions. Electroencephalogram (EEG) is considered a valuable physiological source of information for classifying emotions. However, it has complex and chaotic behavior.

Methods: In this study, an attempt is made to extract important nonlinear features from EEGs with the aim of emotion recognition. We also take advantage of machine learning methods such as evolutionary feature selection methods and committee machines to enhance the classification performance. Classification performed concerning both arousal and valence factors.

Results: Results suggest that the proposed method is successful and comparable to the previous works. A recognition rate equal to $90 \%$ achieved, and the most significant features reported. We apply the final classification scheme to 2 different databases including our recorded EEGs and a benchmark dataset to evaluate the suggested approach.

Conclusion: Our findings approve of the effectiveness of using nonlinear features and a combination of classifiers. Results are also discussed from different points of view to understand brain dynamics better while emotion changes. This study reveals useful insights about emotion classification and brain-behavior related to emotion elicitation.

Keywords: Emotion Recognition, Phase Space Reconstruction, Nonlinear EEG Analysis, Committee Machine, Evolutionary Feature Selection.
\end{abstract}

Citation: Zangeneh Soroush M, Maghooli K, Setarehdan SK, Motie Nasrabadi A. Emotion classification through nonlinear EEG analysis using machine learning methods. Int Clin Neurosci J. 2018;5(4):135-149. doi:10.15171/icnj.2018.26
*Correspondence to

Assistant Professor, Department of Biomedical Engineering, Science and Research Branch, Islamic Azad University, Tehran, Iran.

Tel: +989122993212

Email: k_maghooli@srbiau.ac.ir

Published online 20 December 2018

\section{Introduction}

Indeed, emotions play a crucial role in our daily life and communication including human-human and humanmachine interaction. According to recent studies, emotions and emotion recognition have used in numerous areas such as education, health, decision making, robotics, rehabilitation, driving assistance, and many more. Emotions might be considered seemingly simple at first glance. However, they are still unknown regarding the origin and their mechanisms. Emotion regulation considered one of the most complex brain processes since it ultimately relates to time, location, context, culture, and language. Our knowledge of emotions is limited, and there is no clear definition of the different feelings one can experience. Researchers from a large number of interdisciplinary fields such as psychology, biomedical engineering, robotics, and many more have tried to develop and enhance emotion classification systems with the aim of analyzing, interpreting and detecting human feelings. Despite considerable attempts in this area, emotions and emotion recognition have remained largely unexplored, ${ }^{1,2}$ which motivate us to study emotions in this paper.

There are 2 major ways to describe emotions called discrete and dimensional models. In the discrete view, emotions can categorize in previously defined states such as fear, sadness, happiness and so on. However, in the dimensional perspective emotions are described by at least 2 factors: arousal and valence. Human feelings can represent onto a $2 \mathrm{D}$ plane where there is $\mathrm{x}$-axis (valence) and y-axis (arousal). In some studies, it mentions that the dimensional model can better represent emotional states than the discrete one. ${ }^{3}$ Therefore, the dimensional point of view applied in this study. Figure 1 shows the arousalvalence plane and also some basic emotions on it. As can be seen, the arousal and valence factors vary from positive to negative and calm to exciting, respectively. Emotional states can easily define in 4 different quadrants (Q1Q4) including high arousal-high valence (HAHV), high arousal-low valence (HALV), low arousal-low valence (LALV) and low arousal-high valence (LAHV).

In recent years, several modalities such as facial 


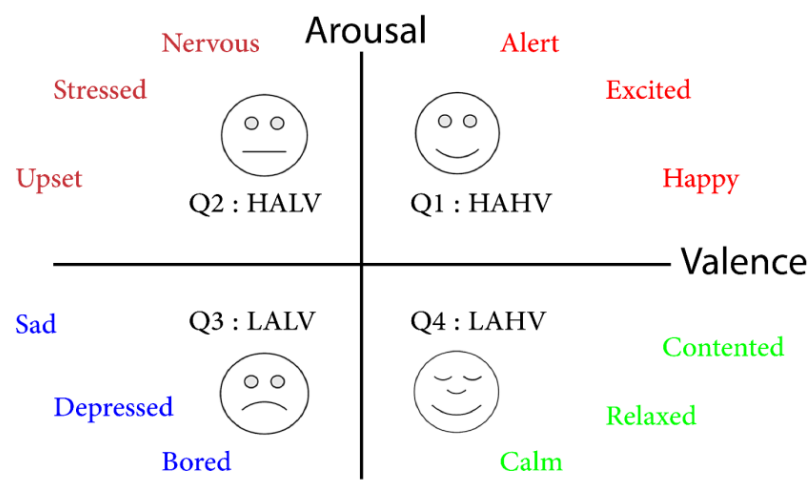

Figure 1. Arousal-Valence Plane for Emotions With Some Basic Emotions on it. The horizontal and vertical axes describe valence and arousal respectively.

expression, speech and body gestures have been employed to classify emotions. Though sometimes accurate, the reflected emotions are usually subjective. Although these modalities have produced significant results, they are sensitive to social masking. These restrictions unlock the way to recognizing emotions through physiological signals. On the contrary, physiological signals such as electroencephalogram (EEG), electrocardiogram (ECG), skin temperature and galvanic skin response (GSR) are affected by emotions and controlled by the central nervous system (CNS). Based on, ${ }^{4}$ feelings originate from the brain's response to physiological changes and cannot control by individuals. So these physiological emotion cues can truly reflect emotional states. Simple to record and cost-effective, EEG-based emotion recognition has been attracting a great deal of attention from researchers working in different fields in recent years. EEG seems to be less invasive, with better time resolution than the other physiological measures. In general, EEGs have been broadly employed to study brain activity related to effective responses. EEG has been used in many emotion recognition systems as it can reflect emotions more precisely and reliably at the higher time, spatial and frequency resolution. Also, EEG can continuously detect changes in emotions. ${ }^{5,6}$ In this study, we try to analyze EEG in order to classify emotions accurately.
Thanks to the advent of emotion recognition methods, it is now possible to record and analyze EEG in natural settings. This development has produced a new trend that integrates human-machine interfaces (HMIs) with emotional factors. ${ }^{7}$ Physicians and psychologists are now able to diagnose and treat people's mental disorders like depression, autism, and many more. Employing these interfaces. Figure 2 illustrates the emotional braincomputer interface system. Emotional brain-computer interfaces include 5 main steps. First, individuals exposed to before-designed stimuli based on a protocol. EEGs recorded, and then the raw signals will be preprocessed to eliminate noise and artifacts. Some significant features are then extracted and fed into a classification model. A classifier (or maybe a combination of classifiers) will train according to the selected attributes. After recognizing an individual's current emotions, feedback can be carried out to react to the users. This procedure might be online or offline. ${ }^{7}$ We can conclude that emotions can be reflected and recognized through EEG processing, which suggests the considerable importance of EEG-based emotion recognition systems.

On the other hand, there are several reasons why EEGbased emotion classification is challenging, like unclear boundaries for emotions and subjective opinions towards human feelings. Besides, there is no 'ground truth' for emotions to label EEGs, and also EEG patterns vary from person to person in different emotions. Moreover, emotion classification systems are mostly inaccurate and subject-dependent. It means that finding stable features and designing precise models with high classification performance is still important. Nonlinear analysis is an appropriate way to know more about complex systems like brain and emotions. Considering the chaotic and nonlinear behavior of EEG, simple time and time-frequency analyses are not able to fully extract and represent EEG features and behavior. ${ }^{8}$ Employing nonlinear models for emotions and feelings is not just about EEG processing. Jafari et al used chaotic models to simulate love, hate, etc. ${ }^{9}$ In this study, we focus on reconstructing EEG in the phase space and extracting interactions between components in different emotions. Global information related to

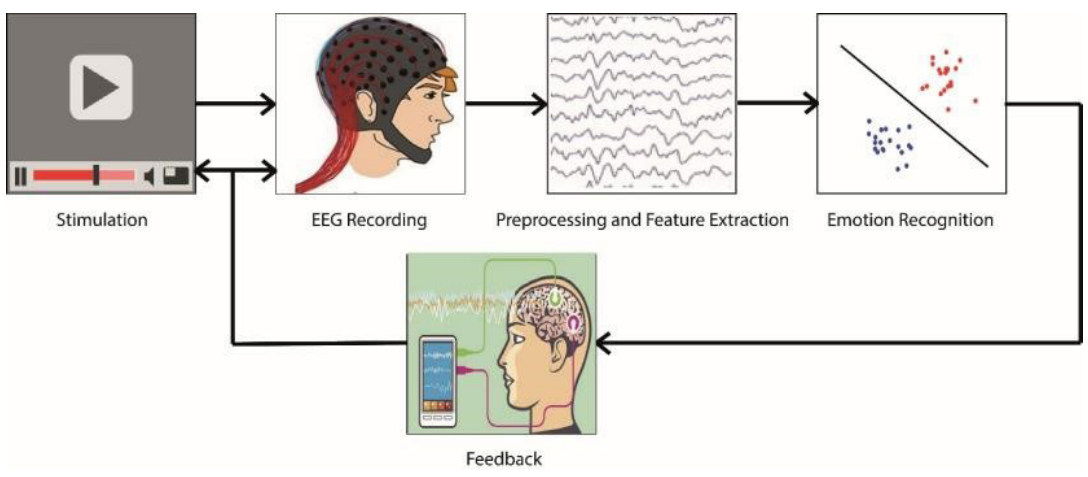

Figure 2. Emotional Brain-Computer Interface. 
emotional states also achieved in this work. Several studies have been conducted in affective computing through EEG nonlinear analysis with the aim of emotion recognition. Some surveys introduce EEG-based emotion classification in detail. Almost all studies in this field have claimed that chaotic features are quite effective in emotion recognition. ${ }^{10-12}$

In recent years, several articles including engineering approaches to automatic emotion classification using EEG nonlinear characteristics (chaotic behavior) and pattern recognition methods have been published. ${ }^{13}$ Nonlinear analysis has employed in numerous fields such as medicine and biomedical engineering over the past few years. In particular, the nonlinear analysis approaches are effectively used to investigate EEGs and the dynamics of the complex underlying system, and it is quite clear that the EEGs exhibit significant chaotic behavior. Non-linear analysis helps us to represent and recognize the irregular behaviors which exist in the system. Numerous nonlinear attributes such as embedding dimension (ED), fractal dimension (FD), correlation dimension (CD), detrended fluctuation analysis (DFA), approximate entropy (ApEn), multi-scale entropy (MsEn), Shannon entropy (ShEn), permutation entropy (PerEn), largest Lyapunov exponent (LLE), Lempel-Ziv complexity (LZC), recurrence quantification analysis (RQA) and Hurst exponent $(\mathrm{H})$ have been widely employed to characterize EEG. ${ }^{13-19}$ These chaotic features are more likely to reflect and represent realistic information about the physiological state of the CNS which is inherently complex and nonlinear.

Due to the importance of emotion recognition through nonlinear analysis, we review some significant studies in this area. EEG is claimed to be able to provide more information related to emotions than other physiological variables. ${ }^{20}$ Although emotion recognition through EEGs seems complicated because of the reasons as mentioned earlier, some recent works have managed to discern different emotional states from EEG. EEG has characterized from both the linear and nonlinear points of view. However, more relevant insights have provided by nonlinear analysis. ${ }^{21}$ Some studies have reviewed recent studies related to EEG-based emotion classification through nonlinear analysis. ${ }^{12,22-24}$

Table 1 represents some previous emotion recognition

Table 1. Previous Studies on Emotion Recognition Using Nonlinear EEG Analysis

\begin{tabular}{|c|c|c|c|c|c|c|c|c|}
\hline Database & Stimuli & \#Chan. & \#Part & Features & Classifier & Emotion States & $\begin{array}{c}\text { Classification } \\
\text { Accuracy }\end{array}$ & Ref. \\
\hline DEAP & Video clips & 32 & 32 & $\begin{array}{l}\text { CD, FD, RQA, } \\
\text { ApEn }\end{array}$ & $\begin{array}{l}\text { Dempster-Shafer theory } \\
\text { of evidence }\end{array}$ & $\begin{array}{l}\text { Arousal-valence plane } \\
\text { (4 classes) }\end{array}$ & $91 \%$ & 25 \\
\hline- & Music & 19 & 5 & $\mathrm{CD}$ & $t$ test & $\begin{array}{l}\text { Positive and negative } \\
\text { valence }\end{array}$ & - & 8 \\
\hline DEAP & Video clips & 32 & 32 & CD, FD, SaEn, ApEn & Self organization map & $\mathrm{HA}, \mathrm{HV}, \mathrm{LA}$, and LV & $65 \%$ & 26 \\
\hline- & Video clips & 19 & 40 & $\begin{array}{c}\text { Bispectrum, Power Spectrum, } \\
\text { Wavelet, ApEn, H }\end{array}$ & SVM & $\mathrm{HA}, \mathrm{HV}, \mathrm{LA}$, and LV & $60 \%$ & 27 \\
\hline- & Pictures & 54 & 5 & Statistical Features, CD & $\begin{array}{l}\text { Quadratic discriminant } \\
\text { analysis }\end{array}$ & $\begin{array}{l}\text { Positively excited, } \\
\text { negatively excited, } \\
\text { and calm }\end{array}$ & $66 \%$ & 28 \\
\hline- & Video clips & 62 & 6 & $\begin{array}{c}\text { Wavelet Transform, Power } \\
\text { Spectrum, ApEn, ED, H, FD, } \\
\text { CD }\end{array}$ & SVM & $\begin{array}{l}\text { Positive and negative } \\
\text { emotions }\end{array}$ & $85 \%$ & 29 \\
\hline DEAP & Video clips & 32 & 32 & SaEn & SVM & $\begin{array}{c}\text { Positive and negative } \\
\text { emotions }\end{array}$ & $80 \%$ & 30 \\
\hline- & None & 19 & 45 & $\begin{array}{c}\text { DFA, Power spectrum, FD, } \\
\text { CD, LLE, }\end{array}$ & $\begin{array}{l}\text { Linear discriminate } \\
\text { analysis }\end{array}$ & Depression & $90 \%$ & 31 \\
\hline- & Video clips & 62 & 20 & $\begin{array}{l}\text { Spatial Filtering, Wavelet, } \\
\text { Time-Frequency Analysis }\end{array}$ & $\begin{array}{l}\text { Linear discriminate } \\
\text { analysis, KNN }\end{array}$ & $\begin{array}{l}\text { Happy, surprise, fear, } \\
\text { disgust, and neutral }\end{array}$ & $84 \%$ & 32 \\
\hline- & Music & 14 & 12 & FD & SVM & Arousal-valence plane & $87 \%$ & 33 \\
\hline- & Pictures & 19 & 10 & LZC & SVM & Arousal-valence plane & $83 \%$ & 34 \\
\hline \multirow[t]{2}{*}{-} & Audio & 14 & 5 & $\begin{array}{l}\text { Statistical Features, FD, } \\
\text { Power Spectrum }\end{array}$ & SVM & $\begin{array}{l}\text { Pleasant, happy, } \\
\text { frightened and angry }\end{array}$ & $86 \%$ & 35 \\
\hline & Audio & 14 & 12 & FD & SVM & $\begin{array}{c}\text { Positive and negative } \\
\text { emotions }\end{array}$ & $85 \%$ & 36 \\
\hline- & Music & 19 & 30 & CD, H, LLE, ApEn & ANOVA & Different mental states & - & 37 \\
\hline- & Audio & 7 & 10 & DFA & ANOVA & Different mental states & - & 38 \\
\hline DEAP & Video clips & 32 & 32 & RQA & $\mathrm{KNN}$ & $\begin{array}{l}\text { Arousal, Valence, and } \\
\text { Liking }\end{array}$ & $68 \%$ & 39 \\
\hline
\end{tabular}

Abbreviations: DEAP, database for emotion analysis using physiological signals; SVM, support vector machine; KNN, K nearest neighbor;ANOVA, analysis of variance; CD, correlation dimension; FD, fractal dimension; ApEn, approximate entropy; ROA, recurrence quantification analysis; SaEn, sample entropy; HA, high arousal; HV, high valence; HA, high arousal; LV, low valence: H, Hurst exponent; DFA, detrended fluctuation analysis; ED, embedding dimension; LLE, largest Lyapunov exponent. 
studies employing nonlinear EEG analysis. ${ }^{8,25-39}$ As it can infer from this Table, emotions can distinguish through nonlinear features. In a few studies, researchers focus on both arousal and valence values. In most cases, the discrete model of emotions considered. It suggests that there is still limited knowledge in extracting and selecting significant nonlinear features correspondent to arousal and valence. Some studies do not report classification accuracy as they mainly focus on the class separability of nonlinear features and also EEG complexity analysis related to emotional changes. ${ }^{8,37,38}$ For example Hoseingholizade et al reported that $\mathrm{CD}$ decreased during emotional elicitation in comparison with the rest state. ${ }^{8}$ These studies approve of significant shifts in nonlinear attributes as a biological response to emotional changes. It is worth mentioning that a fair comparison cannot draw since these studies have applied different experimental settings. However, some basic points can infer without over-generalization. Classification accuracy is commensurate with the number of EEG channels. More active EEG channels result in higher recognition performance. Support vector machine (SVM) is broadly utilized in recent studies as it can better classify emotions. Nonlinear features seem to be effective regarding emotion recognition. Considering nonlinearity which exists in the brain responses to emotions, it is imperative to employ nonlinear features as they seem to be more reliable to describe emotional states precisely. These features are more successful as they are more stable and can better reflect emotional changes, which is a complex process, in comparison with statistical, frequency and time-frequency analysis. The linear methods (like power spectral density, Fourier analysis, etc) lack the complex components of the non-stationary, chaotic EEGs and rely on a rough estimation. ${ }^{35,38,39}$

Motivated by the advantages of employing nonlinear features and chaotic analysis, we set out to classify emotions through EEG analysis. We choose audio-visual stimuli to induce emotions as it has employed in numerous emotion recognition studies. Figure 3 shows the block diagram of the proposed method. EEGs are first recorded and then undergo the preprocessing step. Noise reduction and artifact removal are the main part of preprocessing. EEG phase space reconstructed, and proposed features extracted from clear EEGs. In this paper, an attempt is made to quantify the phase space and its characteristics. Extracted features statistically evaluated, and the most significant ones fed into the classification process. We employed 3 basic but efficient feature selection methods based on evolutionary algorithms including a genetic algorithm (GA), particle swarm optimization (PSO) and ant colony (ACO). Three well-known classifiers including multi-layer perceptron (MLP), K-nearest neighbor (KNN) and SVM are employed. Samples classified according to arousal and valence. Some features can describe arousal and some features can better discriminate valence. Since these 2 factors are important while studying emotions,

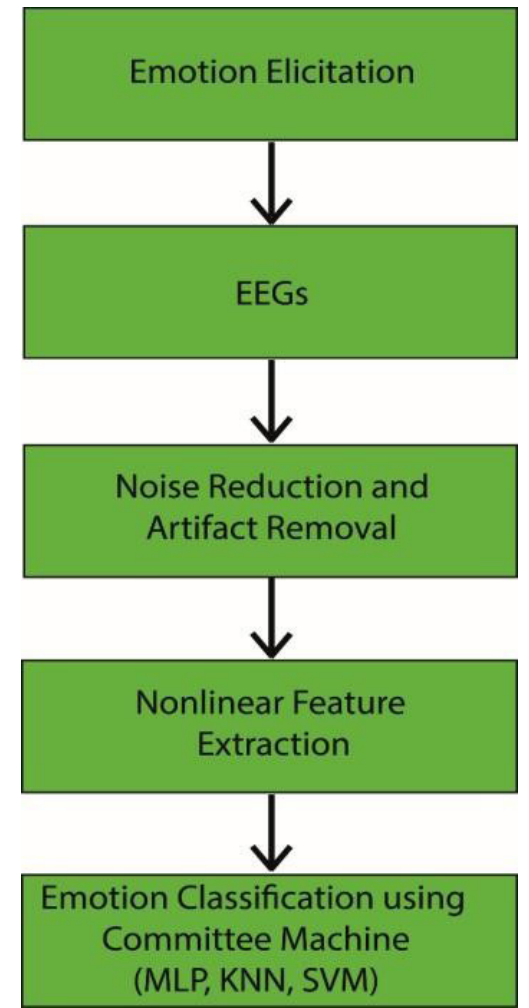

Figure 3. The Block Diagram of the Proposed Method.

the proposed features analyzed regarding both arousal and valence classification. The committee machine using voting procedure is also applied to increase the classification performance. Ten-fold cross-validation is performed, and results reported. Results show that the proposed method is effective regarding both arousal and valence classification. Selected features and brain lobes analyzed for class separability and average activation over brain regions represented and discussed. Computation cost is quite low and comparable to previous studies.

\section{Materials and Methods}

Dataset

Emotion elicitation plays a crucial role in emotionrelated studies. Designing efficient and reliable emotion elicitation and protocols are critical. There are several ways to stimulate emotions such as images, video clips, memories, music, etc however, based on previous works the audio-visual elicitation has its advantages. ${ }^{40-43}$ Since videos contain both scene and audio, individuals are more exposed to real and strong emotional changes. Therefore, we employed video clips to stimulate participants' feelings. We chose 20 videos with the approximate length of 2 minutes from Iranian movies. There are 4 groups of emotions based on the arousal-valence plane. We decided to allocate 5 videos to each emotional quadrant. Video clips were selected accurately and precisely to have the most highlighted scenes related to the correspondent feeling. An investigation conducted employing some 
questionnaires from 10 volunteers who did not participate in the experiment to assure the effectiveness of the selected video clips as the elicitors. Then 20 healthy males and 20 healthy females, aged between 22 and 30 (mean = 26.13 and $\mathrm{SD}=2.79$ years), enrolled in the experiment. All participants confirmed their normal vision and hearing. They also reported no history of mental diseases. Volunteers went through the Eysenck Personality Questionnaire (EPQ) ${ }^{44}$ in order to assessed regarding their personality. This test categorized individuals in 3 independent dimensions including Extraversion/ Introversion, Neuroticism/Stability, and Psychoticism/ Socialization. We selected just extraverted subjects as they can express their exact feelings immediately.

Potential volunteers were notified about the experiment and also basic definitions of emotions, arousal, and valence. They read and signed the consent forms and paid for their cooperation. Some information such as their academic degree, age and sex were also recorded. Each enrolled in 4 different sessions to complete the experiment in each session, 5 videos have shown to them.

Participants were asked to sit comfortably and observe the videos. They were seated approximately $1 \mathrm{~m}$ from the screen. They also claimed not having had caffeinated or alcoholic beverages before the test. Video clips presented in a random order and each clip lasted 2 minutes.
Figure 4 illustrates the experiment scene, recorded EEG channels, self-assessment procedure, and the protocol. The experiment was carried out in a quiet place, and each video clip was preceded by a 5s blank screen notifying the participants about the start of the video. After filling in the self-assessment for 1 minute, an inter-trial interval (30 seconds) of blank screen elapsed between movie clips for emotion resumption.

The experiment performed in the morning and EEG recorded at a sampling frequency of $256 \mathrm{~Hz}$ employing 19 active $\mathrm{Ag} / \mathrm{AgCl}$ sintered ring electrodes based on the international 10-20 system referenced to A1 and $\mathrm{A} 2$ electrodes and grounded to $\mathrm{FPz}$ electrode. Each session consisted of a 5-second initial baseline recording. Impedances checked below $10 \mathrm{k} \Omega$. The EEG was recorded with a resolution of 12 bits/sample.

At the end of each trial, participants were asked to fill in the forms and express their feelings toward the video in one minute. They performed the self-assessment of the level of arousal and valence from 1 to 9. Participants registered their opinions toward each video clip using the self-assessment manikins (SAM). ${ }^{45}$ They were also asked to mark the approximate position of their feelings in the arousal-valence plane and write 2 words to describe them. There is a $5 \mathrm{~s}$ baseline recording before each video clip, one minute for self-assessment and 30s for rest in one
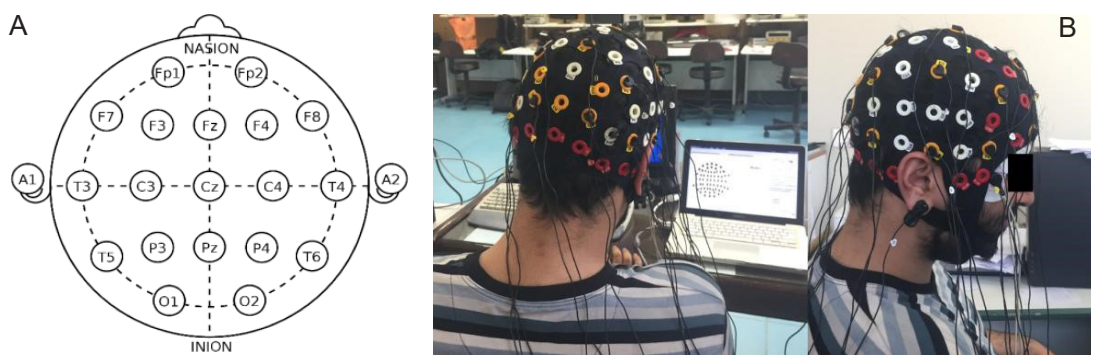

C
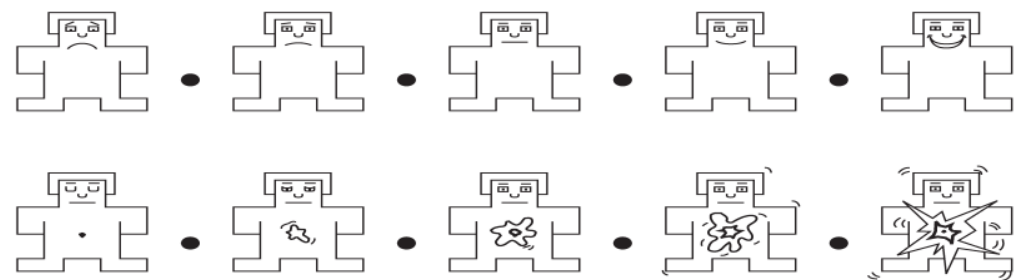

Your feeling in the discrete emotion model:

Arousal value for the video clip: $\quad$ Valence value for the video clip:

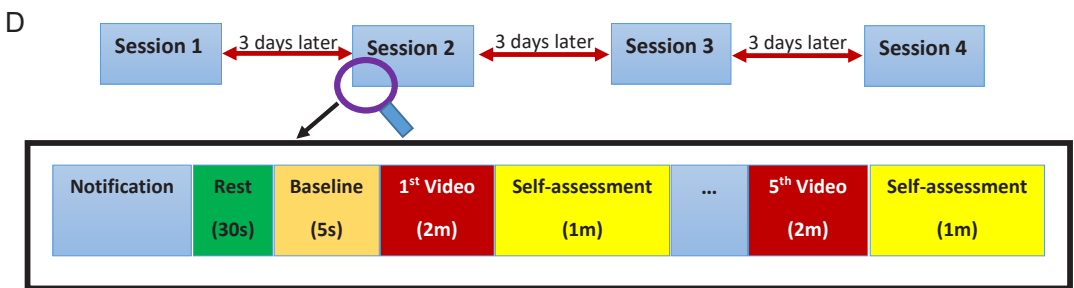

Figure 4. (A) Electrodeposition Based on the 10-20 International System. (B) The Experiment Scene. (C) A Sample for the Self-assessment Form (D) the Protocol of the Experiment. 
trial. The experimenter monitored EEGs, participants, and their assessment. Table 2 represents brief information about this database. We tried to consider all the aspects and design an appropriate experiment close to new and related databases.

Due to the complex behavior of EEG signals, time intervals do not need to be constant. Therefore estimating the best length to window EEGs is of importance. Soroush et $\mathrm{al}^{74}$ proposed a new method to identify the appropriate time intervals to window EEGs based on fractals properties. We compute the relative values of CD and FD for the rest mode. Fluctuation of more than $10 \%$ taken as a new event and a new time interval. EEG signals are windowed and reconstructed in phase space, and then nonlinear features are extracted. These features are quite well-known, and numerous studies have employed them so far. For the sake of space just the features, abbreviations and correspondent references are reported in Table 3. All of the mentioned features categorized as nonlinear time series analysis, and in the previous section, it pointed out that several studies have reported the effectiveness of these attributes while studying emotions through EEG.

\section{Feature Selection}

Feature selection is a process in which an attempt is made to determine a subset of features from the original set of features based on the criterion of feature selection. A large number of features usually results in long processing time and lower classification performance. Considering the number of EEG channels and features, it should note that some feature selection methods must be utilized to reduce the number of features. Inspired by evolutionary approaches, we decided to employ $\mathrm{GA}^{, 75} \mathrm{PSO}^{76,77}$ and $\mathrm{ACO}^{78}$ to optimize the feature selection problem. Mutual information and correlation analysis are also employed to avoid redundancy between selected features. Selected features normalized before classification.

GA is a computational model which selects a set of candidate solutions (chromosomes). All candidates make a population together. The algorithm finds the best solution through some iterations which are called generations. In each generation, 2 chromosomes are considered to be the parents and reproduce the next generation. All chromosomes are sorted based on a fitness function (i.e., $\mathrm{k}$ nearest neighbor in this study). It should note that mutation also happens in each generation according to a very low probability. ${ }^{75}$

Inspired by the nature of flying birds, PSO solves the optimization problems. Again, a set of solutions considered, and each solution has taken as a particle with an absolute velocity. PSO finds the optimum regions of complex search through the population of particle swarms. The velocity of each particle determines the step size of each dimension. In a feature selection problem, PSO considers features as points in the space. A particle put in each point (the subset of features). The particles
Table 2. Brief Information About the Experiment

\begin{tabular}{|c|c|}
\hline Emotion elicitation & Video clips \\
\hline Number of participants & 40 people, $50 \%$ female, aged: $22-30$ \\
\hline Number of video clips & 20 selected 2 -minute video clips \\
\hline Recorded signals & 19-channel $256 \mathrm{~Hz}$ EEG \\
\hline Ratings & Arousal and valence \\
\hline Rating scale & $\begin{array}{l}\text { SAM, 2-word expression, approximate } \\
\text { position in the arousal-valence plane and } \\
\text { continuous scale of } 1 \text { - } 9\end{array}$ \\
\hline Signal length & Baseline: $5 \mathrm{~s}$, elicitation: $2 \mathrm{~m}$, rest: $30 \mathrm{~s}$ \\
\hline
\end{tabular}

Table 3. Extracted Features in This Work and Related Studies

\begin{tabular}{|c|c|c|}
\hline Feature Description & Abbreviation & Ref. \\
\hline Correlation dimension & CD & 63 \\
\hline Fractal dimension & $\mathrm{FD}$ & 64,65 \\
\hline Largest Lyapunov exponent & LLE & 63 \\
\hline Embedding dimension & $\mathrm{ED}$ & 64 \\
\hline Average time lag & ATL & 64 \\
\hline Recurrence rate & $\mathrm{RR}$ & $66-69$ \\
\hline Determinism & DET & $66-69$ \\
\hline Laminarity & LAM & $66-69$ \\
\hline Maximum length of vertical structures & MLV & $66-69$ \\
\hline Recurrence time of first type & RT & $66-69$ \\
\hline Average diagonal line length & $\mathrm{L}$ & $66-69$ \\
\hline Maximum length of diagonal structures & MLD & $66-69$ \\
\hline Transitivity & $\mathrm{T}$ & $66-69$ \\
\hline Entropy & ENT & $66-69$ \\
\hline Permutation entropy & PeEn & $70-72$ \\
\hline Approximate entropy & ApEn & $70-72$ \\
\hline Differential entropy & DiEn & 70,71 \\
\hline Sample entropy & SaEn & 70,71 \\
\hline Shannon entropy & ShEn & 72 \\
\hline Log energy entropy & LeEn & 72 \\
\hline
\end{tabular}

then fly to find the best position and meanwhile they interact with each other. Finally, they converge to the best and optimal position which is the optimum feature subset with the highest classification accuracy. ${ }^{76,77}$

In ant colonies, a randomly moving ant decides to follow the path marked by pheromone, and then this ant releases some pheromone to intensify the importance of that path. So the probability of each path can be determined by the number of ants which are following it. This positive feedback results in solving an optimization problem in which artificial ants are employed to build solutions. The fitness value is in proportional to the amount of pheromone. In ACO-based feature selection, many artificial ants equal to the original features are employed to accumulate pheromones and construct sequential solutions. The best ants are selected and fed into the next iteration. In each iteration, ants decide on one of the solutions based on local heuristics and prior knowledge. ${ }^{77,78}$ 
Classification

Three basic but efficient classifiers employed in order to make an appropriate framework to compare the results. MLP, KNN and SVM used as classification models in the current work. Each classifier solves the recognition problem in a different approach - these 3 models combined through the voting algorithm. In this method, the final decision is made based on the maximum vote for the classes.

Complementary information from different classifiers can lead to higher accuracy. The weighted linear combination of voters (classifiers) can be considered a way to improve the quality of final classification. Several algorithms such as $\mathrm{MinCq}^{79}$ propose a method to learn a weighted majority vote. This method based on a quadratic program that minimizes the generalization bound and ensures learning of the classification model according to theoretical guarantees. We suppose that this mixture can help us classify emotions more precisely.

\section{Results and Discussion}

EEGs as a response to emotion elicitation through watching video clips, are recorded and then preprocessed employing SOBI to separate components and SWT to suppress noise and artifacts. Clean EEGs are windowed, and phase space reconstructed for each channel. As mentioned before, nonlinear features extracted from all EEG channels. Evolutionary algorithms utilized for feature selection. Significant features fed into the classification step. It is worth mentioning that all of the data divided into 3 parts for feature selection, training and testing the proposed model. Ten-fold cross-validation is then performed to evaluate the suggested approach.

For the preprocessing step, it should note that biological artifacts mainly arise from 2 significant sources including environmental and physiological signals. Conventional methods like linear filters are not appropriate and effective due to inherent overlap in the frequency domain between artifacts and cerebral activity. BSS approaches have been reported to be successful in extracting EEG sources. Different articles have concluded that independent component analysis (ICA) is the most robust method in artifact elimination but is not very fast concerning time.

\section{Preprocessing}

Unfortunately, in most practical settings EEGs are corrupted by environmental and physiological signals called EEG artifacts. ${ }^{46,47}$ Blind source separation (BSS) methods have been receiving a great deal of attention in EEG processing and artifact suppression since they isolate noise and artifacts into independent components (ICs) using subspace filtering. ${ }^{47}$ Second order blind identification (SOBI) utilizes the original EEG and timeshifted version(s) in order to exploit temporal information and estimate uncorrelated components. Among different BSS based methods, SOBI is reportedly the most effective one while processing EEG. ${ }^{47-56}$ Therefore, we decided to use SOBI in this study to extract EEG sources. EEG sources are visually investigated by experts using temporal, spatial and topographical representations.

A well-known algorithm to suppress artifacts is decomposing artifactual components by wavelet transform. Decomposed sub-bands are denoised by thresholding. ${ }^{48-50}$ Several studies have suggested wavelet with the aim of artifact elimination. ${ }^{57-60}$ The type of wavelet transform varies in each study. It can be a discrete wavelet transform (DWT), continuous wavelet transform (CWT) or stationary wavelet transform (SWT) ${ }^{48-50}$ As it states in, ${ }^{55,59}$ SWT is superior to DWT and CWT in removing biological artifacts. So in this study identified artifactual sources are eliminated using SWT.

It should note that in this step EEGs are also common averaged referenced and high-pass filtered employing EEGLAB. ${ }^{46}$ The cutoff-frequency is $2 \mathrm{~Hz}$. All recordings visually checked and EEGs severely contaminated by EMG and EOG are removed manually. EEGs observed so that no noticeable artifacts or noises exist after the preprocessing step.

\section{Feature Extraction}

All features in this study are related to the phase space of EEGs. Phase space reconstruction plays an important role in the nonlinear analysis and is the key to gaining information about complex signals. An attempt is made to focus on the features extracted from phase space. Suppose that $x(t)$ is a signal with $N$ time samples. We can reconstruct $N-p+1$ vectors in the phase space as:

$X(i)=[x(i+T) x(i+2 T) \ldots x(i+N-(p-1) T)] i=1,2, \ldots, N-(p-1)$ $T$

Where $p$ and $T$, which are the ED and time delay respectively, can be estimated by using the false nearestneighbors algorithm ${ }^{61}$ and the mutual information. ${ }^{62}$ (see Table $3^{63-72}$ ).

Figure 5 shows a sample of corrupted and clean EEG using the proposed preprocessing method. As it can be seen SOBI and SWT make a useful contribution to EEG noise removal. After source separation, different views can be taken into account to remove artifacts. One can set artifactual components to zero which is not very practical since it is entirely possible that neural information (might) leak into these components. So ignoring all artifactual sources might lead to information loss. Although this approach seems to be very simple, it leads to significant distortion in reconstructed EEGs.

Additionally, SWT is translation-invariant which suggests its superiority to DWT while removing biological artifacts. $^{48,49,55,59}$ We employ SWT to denoise detected artifactual components and to prevent data loss since there is always information leakage to artifact components while using BSS methods. SWT can maintain cerebral activity and a large extent, reject artifacts too. Remained 

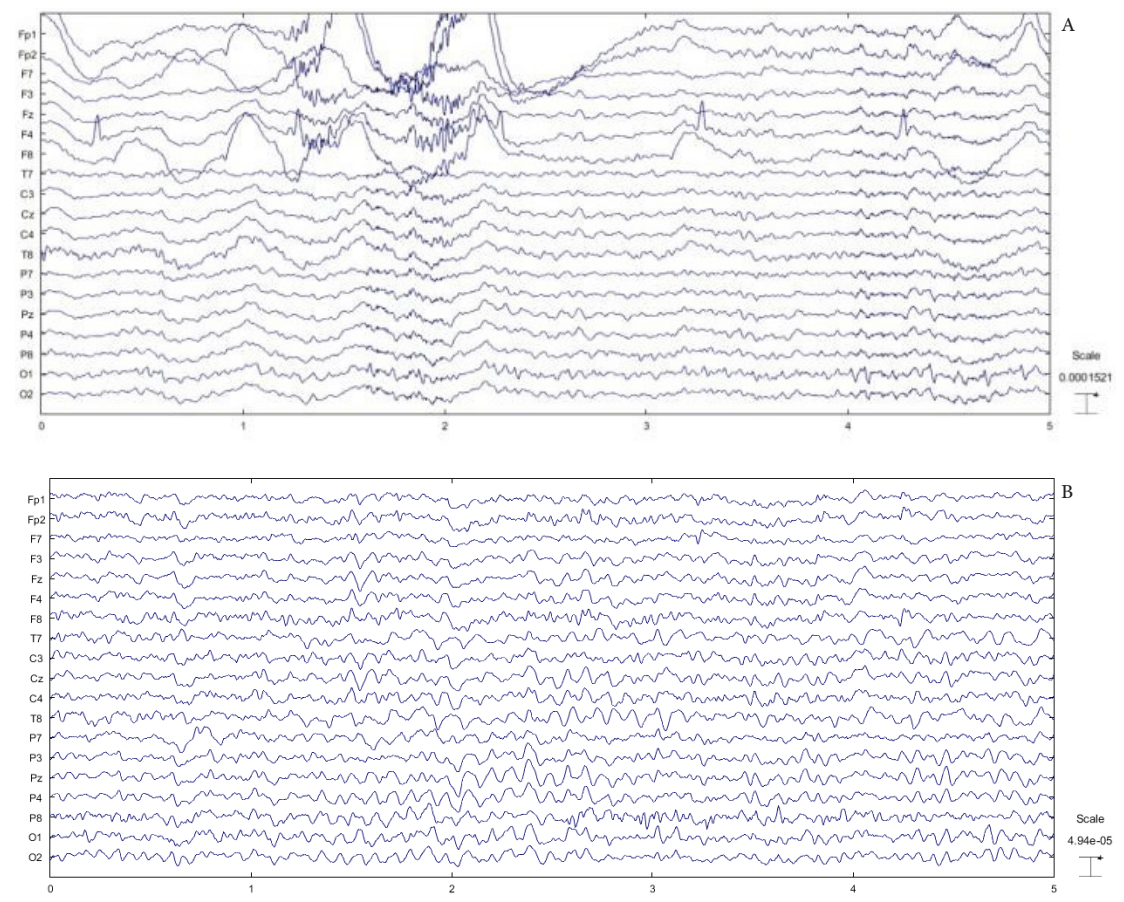

Figure 5. (A) Contaminated and (B) Preprocessed EEG Using the Suggested Method.

components are used to reconstruct clean EEG.

Noise-free EEGs are windowed using changes in 2 important parameters including CD and FD. It assumed that emotional stimuli lead to considerable changes in EEG behavior and information. As mentioned before, this approach is found practical in previous studies such as. ${ }^{74}$ The emotional changes result in information creation, bifurcation in EEGs and consequently fluctuations in $\mathrm{CD}$ and FD. Not only are these attributes related to the changes in information index and bifurcation but also, they are less sensitive to noise and artifacts in comparison with other features. Nonlinear attributes extracted from windowed EEGs and feature selection performed. Tables 4 and 5 represent the most significant features which are selected by GA, PSO, and ACO. Two-sample $t$ test is also carried out for each method in order to investigate selected features regarding separability for arousal and valence. Ten most significant features, $P$ values, and correspondent EEG channels are reported in Tables 4 and 5 for arousal and valence classification respectively.

As it can see, nonlinear features are quite adequate for arousal and valence classification alike. We also consider the selected features and the average $P$ values for the proposed nonlinear features. These average values can assume as some comparison criteria among the feature selection methods. While GA performs better for arousal, PSO-based feature selection method seems to be more efficient for valence.

It can see that RQA is more successful in arousal-based emotion classification. Some attributes like RR, LAM, and DET are common among the proposed methods. Bahari and Janghorbani have also approved that emotions can classify through RQA. ${ }^{39}$ In contrast, different entropies seem to be more efficient in valence-based emotion

Table 4. Ten Most Significant Features Identified by GA, PSO, and ACO for Arousal Classification

\begin{tabular}{|c|c|c|c|c|c|c|c|c|}
\hline \multicolumn{3}{|l|}{ GA } & \multicolumn{3}{|l|}{ PSO } & \multicolumn{3}{|l|}{$\mathrm{ACO}$} \\
\hline Feature & Channel & $P$ Value $* 10^{-2}$ & Feature & Channel & $P$ Value $* 10^{-2}$ & Feature & Channel & $P$ Value $* 10^{-2}$ \\
\hline $\mathrm{CD}$ & Fp1 & 1.92 & DET & T6 & 2.67 & RT & $\mathrm{F} 4$ & 2.88 \\
\hline LeEn & $\mathrm{T} 3$ & 3.93 & ENT & Fp1 & 3.22 & SaEn & F8 & 3.91 \\
\hline FD & F7 & 2.12 & $\mathrm{FD}$ & P4 & 1.76 & ATL & Fp1 & 2.69 \\
\hline $\mathrm{T}$ & $\mathrm{O} 1$ & 2.47 & MLV & $\mathrm{Fp} 2$ & 4.67 & CD & $\mathrm{Fp} 2$ & 3.47 \\
\hline SaEn & $\mathrm{T} 4$ & 2.56 & $\mathrm{CD}$ & F3 & 1.34 & LLE & T6 & 2.39 \\
\hline LAM & Fp2 & 1.32 & $\mathrm{ED}$ & $\mathrm{O} 2$ & 2.97 & ApEn & F4 & 4.17 \\
\hline LLE & $\mathrm{C} 3$ & 3.15 & LAM & F7 & 2.02 & FD & Fp2 & 3.28 \\
\hline SE & T5 & 4.19 & $\mathrm{RR}$ & Fp2 & 3.17 & $\mathrm{~T}$ & T6 & 4.77 \\
\hline $\mathrm{FD}$ & F3 & 2.93 & LLE & O1 & 4.22 & DET & O1 & 3.14 \\
\hline \multirow[t]{2}{*}{$\mathrm{RR}$} & P3 & 3.81 & MLD & $\mathrm{C} 3$ & 3.63 & $\mathrm{~L}$ & F7 & 4.55 \\
\hline & & 2.84 & & & 2.97 & & Average & 3.52 \\
\hline
\end{tabular}


Table 5. Ten Most Significant Features Identified by GA, PSO, and ACO for Valence Classification

\begin{tabular}{|c|c|c|c|c|c|c|c|c|}
\hline \multicolumn{3}{|l|}{ GA } & \multicolumn{3}{|l|}{ PSO } & \multicolumn{3}{|l|}{ ACO } \\
\hline Feature & Channel & $P$ Value $* 10^{-2}$ & Feature & Channel & $P$ Value $* 10^{-2}$ & Feature & Channel & $P$ Value $* 10^{-2}$ \\
\hline SaEn & Fp1 & 2.27 & ApEn & T6 & 3.34 & ATL & F3 & 3.14 \\
\hline$C D$ & Fp1 & 1.98 & DET & $\mathrm{F} 4$ & 1.86 & ApEn & Fp2 & 3.97 \\
\hline $\mathrm{L}$ & F7 & 4.33 & ApEn & $\mathrm{O} 1$ & 2.99 & $\mathrm{CD}$ & F7 & 1.39 \\
\hline DiEn & F7 & 1.78 & RT & Fp2 & 3.13 & PeEn & T3 & 4.13 \\
\hline MLD & $\mathrm{O} 1$ & 2.42 & LAM & P4 & 4.72 & $\mathrm{RR}$ & $\mathrm{O} 2$ & 3.53 \\
\hline DET & $\mathrm{F} 4$ & 3.16 & $\mathrm{CD}$ & $\mathrm{T} 4$ & 2.73 & ENT & F3 & 2.91 \\
\hline DiEn & $\mathrm{T} 4$ & 4.65 & $\mathrm{ED}$ & $\mathrm{O} 2$ & 3.17 & SaEn & $\mathrm{T} 3$ & 3.42 \\
\hline $\mathrm{L}$ & F3 & 2.63 & ShEn & T6 & 1.02 & RT & Fp1 & 3.64 \\
\hline MLV & $\mathrm{C} 4$ & 3.96 & ATL & $\mathrm{C} 4$ & 2.81 & LAM & P3 & 2.76 \\
\hline \multirow[t]{2}{*}{ LeEn } & $\mathrm{C} 3$ & 4.36 & $\mathrm{RR}$ & Fp1 & 3.79 & FD & F7 & 4.08 \\
\hline & & 3.16 & & & 2.94 & & Average & 3.29 \\
\hline
\end{tabular}

recognition. The usage of entropies in EEG-based emotion recognition has also reported in. ${ }^{27,29,30} \mathrm{CD}$ and $\mathrm{FD}$ have almost the same portions for arousal and valence. In some previous works such as, ${ }^{74}$ it claims that these features are active while classifying emotional states.

To go further, we decided to analyze the results in Tables 4 and 5 in topographic brain maps. Figures 6 and 7 illustrate the separability index in brain lobes for arousal and valence respectively -50 of the most significant features selected for each method and the whole separability index calculated. It can infer that frontal and temporal lobes have the most profound effect. It is claimed that these lobes can truly reflect emotional changes. ${ }^{10,37,74}$ Occipital lobe seems to be significant in comparison with other lobes as it is the origin of neural activity in response to visual stimuli. Take a close look at Figure 6; it can see that frontal lobe is reported to be the most effective lobe for arousal. Besides that, temporal and occipital lobe plays a vital role for valence in Figure 7. Based on Tables 4 and 5 , most selected features are from these mentioned brain

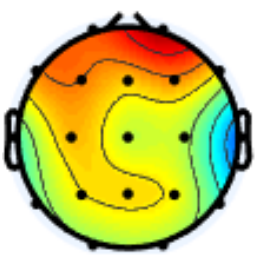

(A)

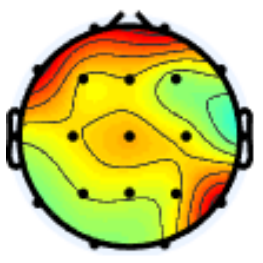

(B)

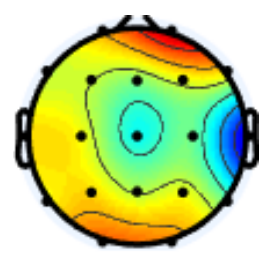

(C)
Figure 6. EEG Channels Separability Index for Arousal Determined by (A) GA, (B) PSO and (C) ACO.

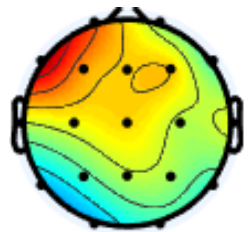

(A)

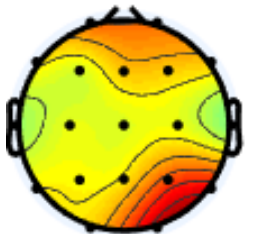

(B)

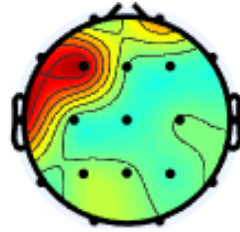

(C)
Figure 7. EEG Channels Separability Index for Valence Determined by (A) GA, (B) PSO and (C) ACO. regions. One can choose Fp1, Fp2, F3, F7, T3, T4, C3, $\mathrm{P} 3, \mathrm{O} 1$ and $\mathrm{O} 2$ as the most influential EEG channels in emotion recognition. By processing only these channels, it is more likely to decrease computation cost.

We are also motivated to compare features in details.

The average rate of presence has calculated among the top 50 features over all the channels for each attribute. The average score reported in Table 6 . This score suggests the contribution that each feature makes and can be employed as a criterion to compare the results. Surprisingly, CD and FD have the same score. It can also realize that RQA has a higher score regarding arousal classification in comparison with entropies. Seemingly, higher scores go to entropies when it comes to valence.

Ten-fold cross-validation is performed to evaluate the proposed method. Then, performance criteria including accuracy (ACC), sensitivity (SEN), specificity (SPC) and precision (PER) calculated. These parameters are defined as shown below:

$$
\begin{aligned}
& A C C=\frac{T P+T N}{T P+T N+F P+F N} \\
& S E N=\frac{T P}{T P+F N} \\
& S P C=\frac{T N}{T N+F P}
\end{aligned}
$$

$P E R=\frac{T P}{T P+F P}$

Where TP, TN, FP, FN stand for true positive, true negative, false positive and false negative respectively.

Table 7 represents the recognition performance for different feature selection and classification methods. All mentioned classification performance parameters reported in percentage.

As it can see, there is a slight difference between recognition approaches. For example, SVM seems to 
Table 6. The Presence of Features Among 100 Selected Attributes

\begin{tabular}{|c|c|c|c|c|c|c|c|}
\hline \multirow{2}{*}{ Features } & \multicolumn{3}{|c|}{ Arousal Classification } & \multicolumn{3}{|c|}{ Valence Classification } & \multirow{2}{*}{ Average Score } \\
\hline & GA & PSO & $\mathrm{ACO}$ & GA & PSO & $\mathrm{ACO}$ & \\
\hline $\mathrm{CD}$ & 9 & 6 & 4 & 7 & 7 & 4 & 6.17 \\
\hline FD & 7 & 5 & 5 & 8 & 5 & 7 & 6.17 \\
\hline LLE & 4 & 8 & 5 & 5 & 2 & 5 & 4.83 \\
\hline ED & 3 & 5 & 4 & 3 & 2 & 3 & 3.33 \\
\hline ATL & 5 & 9 & 5 & 5 & 5 & 4 & 5.50 \\
\hline$R R$ & 7 & 5 & 6 & 5 & 4 & 8 & 5.83 \\
\hline DET & 9 & 2 & 5 & 7 & 7 & 4 & 5.66 \\
\hline LAM & 7 & 7 & 6 & 3 & 4 & 5 & 5.33 \\
\hline MLV & 5 & 5 & 7 & 2 & 8 & 4 & 5.16 \\
\hline RT & 4 & 5 & 6 & 4 & 3 & 5 & 4.50 \\
\hline L & 5 & 4 & 5 & 2 & 4 & 3 & 3.83 \\
\hline MLD & 4 & 5 & 7 & 4 & 6 & 4 & 5.00 \\
\hline $\mathrm{T}$ & 3 & 6 & 2 & 7 & 4 & 5 & 4.50 \\
\hline ENT & 2 & 3 & 6 & 3 & 3 & 4 & 3.50 \\
\hline PeEn & 4 & 5 & 3 & 4 & 7 & 7 & 5.00 \\
\hline ApEn & 8 & 3 & 7 & 8 & 4 & 9 & 6.50 \\
\hline DiEn & 2 & 5 & 4 & 4 & 6 & 3 & 4.00 \\
\hline SaEn & 5 & 3 & 4 & 7 & 6 & 7 & 5.33 \\
\hline ShEn & 4 & 4 & 5 & 9 & 5 & 5 & 5.33 \\
\hline LeEn & 3 & 5 & 4 & 3 & 8 & 4 & 4.50 \\
\hline
\end{tabular}

Table 7. Classification Performance of the Proposed Method Including Different Feature Selection Methods and Classification Models

\begin{tabular}{|c|c|c|c|c|c|c|c|}
\hline \multirow{2}{*}{ Classifiers } & & \multicolumn{3}{|c|}{ Arousal Classification (\%) } & \multicolumn{3}{|c|}{ Valence Classification (\%) } \\
\hline & & GA & PSO & $\mathrm{ACO}$ & GA & PSO & $\mathrm{ACO}$ \\
\hline \multirow{4}{*}{ MLP } & ACC & 76.46 & 75.88 & 71.79 & 75.97 & 74.69 & 72.40 \\
\hline & SEN & 78.10 & 72.63 & 72.57 & 72.73 & 74.27 & 74.90 \\
\hline & SPC & 77.18 & 77.66 & 66.16 & 79.11 & 75.95 & 74.50 \\
\hline & PER & 74.08 & 71.55 & 79.31 & 69.86 & 75.93 & 76.88 \\
\hline \multirow{4}{*}{ KNN } & ACC & 73.61 & 70.95 & 65.73 & 70.17 & 66.62 & 62.01 \\
\hline & SEN & 73.50 & 71.07 & 68.30 & 69.81 & 65.46 & 63.37 \\
\hline & SPC & 74.22 & 69.69 & 65.95 & 70.1 & 66.22 & 65.15 \\
\hline & PER & 73.58 & 70.02 & 67.10 & 69.38 & 67.25 & 64.89 \\
\hline \multirow{4}{*}{ SVM } & $\mathrm{ACC}$ & 86.27 & 81.35 & 76.69 & 82.73 & 77.63 & 79.81 \\
\hline & SEN & 83.32 & 82.65 & 77.11 & 81.09 & 78.25 & 80.05 \\
\hline & SPC & 83.40 & 83.97 & 78.32 & 80.65 & 76.52 & 79.15 \\
\hline & PER & 84.23 & 82.59 & 77.35 & 81.30 & 78.77 & 81.24 \\
\hline \multirow{4}{*}{ Majority Voting 1} & $\mathrm{ACC}$ & 87.42 & 85.73 & 80.25 & 84.58 & 80.64 & 83.16 \\
\hline & SEN & 88.66 & 84.46 & 81.87 & 87.08 & 81.50 & 82.75 \\
\hline & SPC & 86.70 & 83.46 & 80.59 & 87.50 & 81.55 & 82.99 \\
\hline & PER & 85.79 & 85.41 & 82.76 & 86.26 & 82.31 & 80.56 \\
\hline
\end{tabular}

be more successful than 2 other classifiers that proved by previous studies like which preferred SVM. . $7,29,30,33,34^{2}$ Recognition rate is almost higher for arousal in comparison with valence in all classification methods. It has reported in. ${ }^{26,27} \mathrm{GA}$ and PSO are more effective than ACO in this study. We decided to employ the combination of classifiers through the majority voting method in order to enhance the results. Two scenarios can be considered to fuse feature selection methods and classifiers. Figure 8 shows the 2 suggested procedures.

In the first approach, selected features by GA, PSO, and ACO are fed into 3 classifiers separately. We call this fusion majority voting 1 . Considering that each feature selection method views the problem differently, it is reasonable to deduce that the combination of these methods might result in higher accuracy. To go further, we decided to follow the second structure called majority voting 2 in this paper. In this approach, selected features from each method mixed and the most significant ones taken to the classifiers. Classification models are also fused, and a final decision made. Table 8 represents the classification performance for majority voting 2 .

As expected, this combination is more efficient than the previous structures. We also decided to apply the second scenario of emotion recognition to classify data into 4 groups including HAHV, HALV, LAHV and LALV. The 


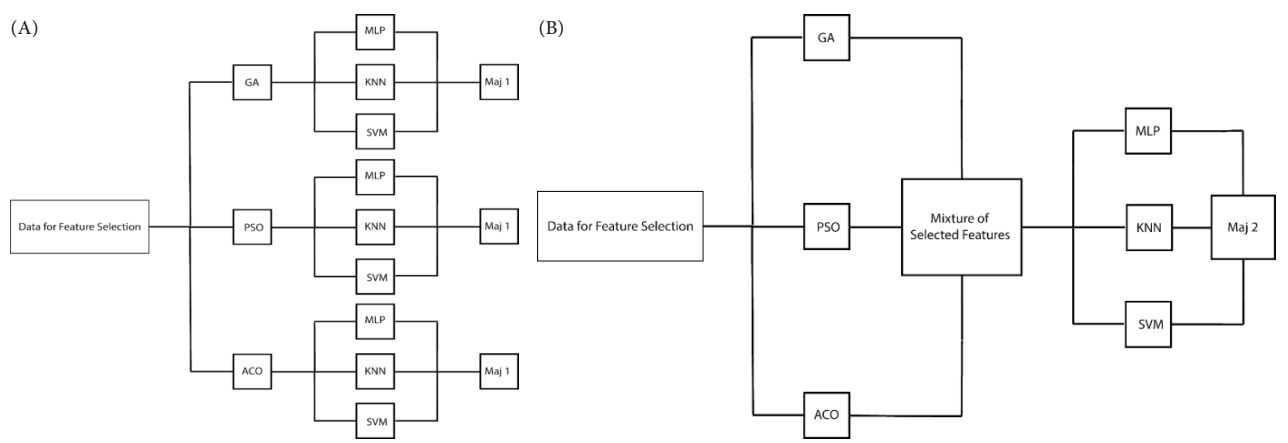

Figure 8. The 2 Suggested Approaches for Combining Feature Selection and Classification Methods. (A) Majority Voting 1 and (B) Majority Voting 2.

Table 8. Classification Performance of Majority Voting 2

\begin{tabular}{lllllllll}
\hline & \multicolumn{4}{l}{ Arousal Classification (\%) } & \multicolumn{4}{c}{ Valence Classification (\%) } \\
\hline \multirow{2}{*}{ Majority Voting 2 } & ACC & SEN & SPC & PER & ACC & SEN & SPC & PER \\
& 91.83 & 89.13 & 91.12 & 89.41 & 90.68 & 88.22 & 91.20 & 89.91 \\
\hline
\end{tabular}

classification accuracy is $83.29 \%$ for the 4 -class emotion recognition. Since arousal and valence are considered 2 major factors indicating emotions, several previous studies concentrate on classifying emotions into the 4 mentioned classes.

In addition, the database for emotion analysis using physiological signals (DEAP $)^{80}$ has been drawn considerable attention from researchers to evaluate their methods. Numerous studies have employed this database such as. . $5,26,30,39$ Thirty-two individuals participated in the experiment, and 40 one-minute video clips shown. Thirty-two EEG channels were recorded and then preprocessed. Similar to our experiment, ratings include arousal and valence. For more information about DEAP, refer to Koelstra et al. ${ }^{80}$

To make a comparison, we decided to apply the proposed method on EEGs from this database and classify emotions into 4 mentioned groups. A classification rate of $84.56 \%$ achieved. Figure 9 illustrates the classification performance of the proposed method and some previous works using DEAP. Taking a closer look at the results, it can conclude that the suggested features, feature selection, and classification approach are quite effective and comparable to other studies. This comparison is made to evaluate the suggested classification framework, however, in the current work the main focus is on our database. We also decided to investigate our recorded EEGs more regarding neural activity and effective brain lobes. Detection of brain lobes associated with emotional states is a matter of immense importance in neuroscience. The average activity for brain lobes shown in Figure 10.

Neural activity is calculated for EEG components and then averaged over all samples for high arousal (HA), low arousal (LA), high valence (HV) and low valence (LV). SOBI has been employed to extract components

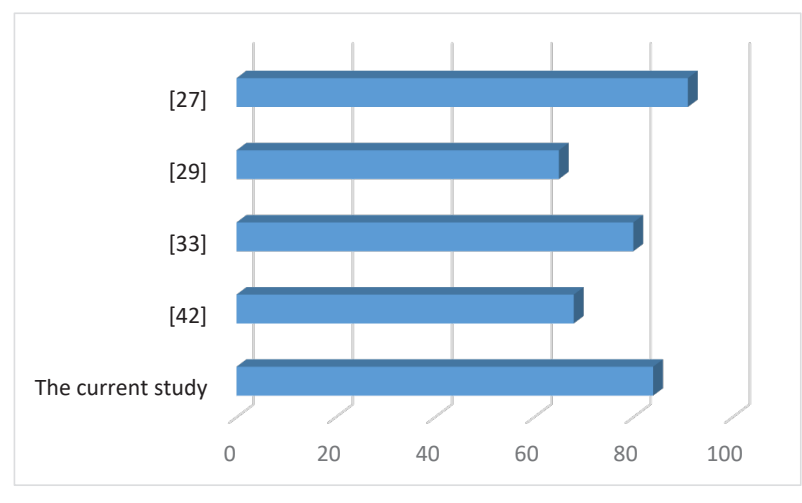

Figure 9. Comparison Between the Proposed Method and Previous Studies Regarding Emotion Recognition Rate Using DEAP.

or to remove artifacts in several studies. Several authors have found SOBI the most reliable and widely used approach. $^{51-53}$ Several toolboxes like EEGLAB have implemented SOBI due to its wide usage and efficiency. SOBI is known as a superior method in comparison with ICA and most BSS methods. Therefore, we decided to use SOBI in this study to extract EEG sources. EEG source separation and topographic mapping performed employing EEGLAB.

Considering the results as mentioned earlier, we can conclude that while frontal and occipital lobes associated with arousal changes, temporal, frontal and occipital lobes correspond to valence. Also, it can see that results support each other in Figures 6 and 7 and Tables 4 and 5. Moreover, a relation between brain regions shown in Figure 10. The physiological meaning of this connection can be investigated in future works using brain connectivity or other computational methods in neuroscience.

Studying effective lobes for arousal and valence not only extends our knowledge but will also be very effective 


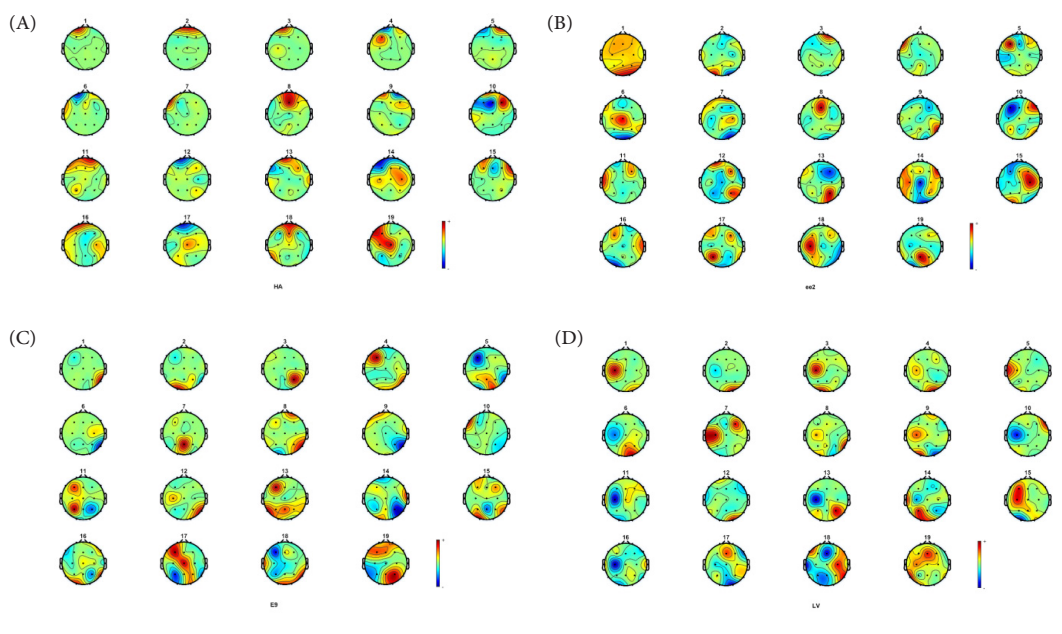

Figure 10. Average Brain Activity Over All Samples in (A) HA, (B) LA, (C) HV and (D) LV.

in real applications in diagnosis and treatment. Since the second scenario of classification (majority voting 2) chosen as the final structure, we consider the 50 most significant features selected by this approach and the proportion of each brain lobe illustrated in Figure 11. Similar to the previous sections, arousal and valence considered, and the brain region shares for each factor calculated. Figure 11 represents the role of each brain lobe in arousal and valence classification. Based on this pie chart, it is clear that frontal and temporal lobes have the most significant impact. Occipital lobe also plays an essential role in arousal classification. Almost onethird of selected features come from the frontal lobe for both arousal and valence. While frontal lobe is the most active region for arousal, temporal lobe seems to be more prominent for valence classification. In contrast to other brain regions, central lobe has almost equal portions in this study that also applies to the parietal lobe. Although previous studies have employed different datasets, some of them like Soroush et $\mathrm{al}^{25}$ approve of almost the same activation patterns for the frontal, temporal and central lobes. As Figure 11 suggests, emotions originate from all brain regions in a nonlinear manner. It can infer that there are nonlinear interactions between these lobes and further studies can concentrate on extracting meaningful information with the aim of understanding emotions better.

All simulations are carried out employing MATLAB (release R2015b) running on Windows 7 Laptop PC with Intel(R) Core(TM) 2 Duo $2.0 \mathrm{GHz}$ processor with $8 \mathrm{~GB}$ RAM. The proposed method is almost computationally intensive, and in further studies, new algorithms could be introduced to make the implementation faster and consequently the proposed method more practical for online emotion recognition.

\section{Conclusion}

In this paper, we introduce a new method to classify

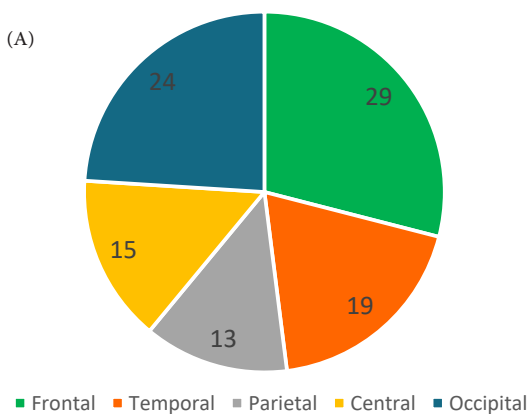

(B)

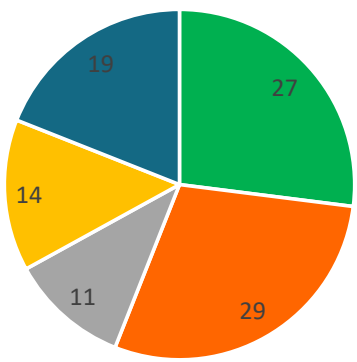

- Frontal - Temporal $₫$ Parietal $\|$ Central - Occipital

Figure 11. The Proportion of Each Brain Region in Feature Presence Among the 50 Most Significant Attributes for (A) Arousal and (B) Valence Classification.

emotions through EEG signals. It reports that nonlinear analysis can better describe complex systems such as emotion regulation. As mentioned before, the brain dynamics in different emotional states reflected by extracting chaotic features like what has employed in this study. We also test evolutionary feature selection methods, and different classification approaches to achieve the highest results. The combination of feature selection methods and classification models (majority voting) results in higher recognition rates. Brain regions associated with emotional states are analyzed, and the results discussed. Like every single study, this paper also has advantages and weak points. More investigation can 
be performed to describe the physiological meaning of the extracted features. Other classification approaches and fusion frameworks can employ in further studies. In the future, researchers can decrease the computation cost and processing time. It is worth mentioning that the advantages of the current study outweigh the drawbacks. This study has prompted us to view and analyze the phase space and nonlinear analysis from different aspects and for our future studies.

\section{Conflict of Interest Disclosures}

The authors declare that they have no conflict of interests.

\section{Ethical Statement}

Not applicable.

\section{Acknowledgments}

The authors are thankful to the Science and Research Branch, Islamic Azad University, Tehran, Iran due to their support.

\section{References}

1. Zheng WL, Zhu JY, Lu BL. Identifying stable patterns over time for emotion recognition from EEG. IEEE Trans Affect Comput. 2017. doi: 10.1109/TAFFC.2017.2712143.

2. Shahnaz C, Shoaib-Bin-Masud, Shafiul Hasan SM. Emotion recognition based on wavelet analysis of Empirical Mode Decomposed EEG signals responsive to music videos. In: 2016 IEEE Region 10 Conference (TENCON); Singapore: IEEE; 2016:424-7. doi: 10.1109/TENCON.2016.7848034.

3. Mauss IB, Robinson MD. Measures of emotion: A review. Cogn Emot. 2009;23(2):209-37. doi: 10.1080/02699930802204677.

4. James W. What is an Emotion? Mind. 1884;9(34):188-205.

5. Zheng W. Multichannel EEG-based emotion recognition via group sparse canonical correlation analysis. IEEE Transactions on Cognitive and Developmental Systems. 2017;9(3):281-90. doi: 10.1109/TCDS.2016.2587290.

6. Jatupaiboon N, Pan-ngum S, Israsena P. Real-time EEGbased happiness detection system. ScientificWorldJournal. 2013;2013:618649. doi: 10.1155/2013/618649.

7. Zheng $\mathrm{WL}$, Lu BL. Investigating critical frequency bands and channels for EEG-based emotion recognition with deep neural networks. IEEE Trans Auton Ment Dev. 2015;7(3):16275. doi: 10.1109/TAMD.2015.2431497.

8. Hoseingholizade S, Golpaygani MRH, Monfared AS. Studying emotion through nonlinear processing of EEG. Procedia Soc Behav Sci. 2012;32:163-9. doi: 10.1016/j. sbspro.2012.01.026.

9. Jafari S, Sprott JC, Golpayegani SMRH. Layla and Majnun: a complex love story. Nonlinear Dyn. 2016;83(1-2):615-22. doi: 10.1007/s11071-015-2351-3.

10. Xu T, Zhou Y, Wang Z, Peng Y. Learning Emotions EEGbased Recognition and Brain Activity: A Survey Study on $\mathrm{BCl}$ for Intelligent Tutoring System. Procedia Comput Sci. 2018;130:376-82. doi: 10.1016/j.procs.2018.04.056.

11. Alarcao SM, Fonseca MJ. Emotions recognition using EEG signals: A survey. IEEE Trans Affect Comput. 2017;PP(99):120. doi: 10.1109/TAFFC.2017.2714671.

12. Zangeneh Soroush M, Maghooli K, Setarehdan SK, Motie Nasrabadi A. A Review on EEG Signals Based Emotion Recognition. Int Clin Neurosci J. 2017;4(4):118-29.

13. Yuvaraj R, Murugappan M, Mohamed Ibrahim N, Sundaraj $\mathrm{K}$, Omar Ml, Mohamad K, et al. Detection of emotions in Parkinson's disease using higher order spectral features from brain's electrical activity. Biomed Signal Process Control. 2014;14:108-16. doi: 10.1016/j.bspc.2014.07.005.

14. McCarthy RA, Warrington EK. Cognitive neuropsychology: A clinical introduction. San Diego: Academic Press; 2013.

15. Stam CJ, Pijn JP, Suffczynski P, Lopes da Silva FH. Dynamics of the human alpha rhythm: evidence for non-linearity? Clin Neurophysiol. 1999;110(10):1801-13.

16. Gao J, Hu J, Tung WW. Facilitating joint chaos and fractal analysis of biosignals through nonlinear adaptive filtering. PLoS One. 2011;6(9):e24331. doi: 10.1371/journal. pone.0024331.

17. Balli T, Palaniappan R. Classification of biological signals using linear and nonlinear features. Physiol Meas. 2010;31(7):90320. doi: 10.1088/0967-3334/31/7/003.

18. Chua KC, Chandran V, Acharya UR, Lim CM. Application of higher order spectra to identify epileptic EEG. J Med Syst. 2011;35(6):1563-71. doi: 10.1007/s10916-010-9433-z.

19. Shen M, Chan FH, Sun L, Beadle PJ. Parametric bispectral estimation of EEG signals in different functional states of brain. IEE Proceedings - Science, Measurement and Technology. 2000;147(6):374-7. doi: 10.1049/ip-smt:20000847.

20. Jenke R, Peer A, Buss M. Feature extraction and selection for emotion recognition from EEG. IEEE Trans Affect Comput. 2014;5(3):327-39. doi: 10.1109/TAFFC.2014.2339834.

21. Valenza G, Lanata A, Scilingo EP. The role of nonlinear dynamics in affective valence and arousal recognition. IEEE Trans Affect Comput. 2012;3(2):237-49. doi: 10.1109/T-AFFC.2011.30.

22. Alcaraz R, Garcia-Martinez B, Zangroniz R, MartinezRodrigo A. Recent Advances and Challenges in Nonlinear Characterization of Brain Dynamics for Automatic Recognition of Emotional States. In: International Work-Conference on the Interplay Between Natural and Artificial Computation 2017 Jun 19. Cham: Springer; 2017:213-22.

23. Garcia-Martinez B, Martinez-Rodrigo A, Alcaraz R, FernandezCaballero A, Gonzalez P. Nonlinear Methodologies Applied to Automatic Recognition of Emotions: An EEG Review. In: International Conference on Ubiquitous Computing and Ambient Intelligence 2017 Nov 7. Cham: Springer; 2017:75465.

24. Soroush MZ, Maghooli K, Soroush PZ, Tahvilian P, Bagherzadeh S. EEG-Based Emotion Recognition through Nonlinear Analysis. 2018;7(78):62-9.

25. Zangeneh Soroush M, Maghooli K, Setarehdan SK, Motie Nasrabadi A. A novel method of eeg-based emotion recognition using nonlinear features variability and Dempster-Shafer theory. Biomed Eng Appl Basis Commun. 2018;30(4):1850026. doi: 10.4015/S1016237218500266.

26. Hatamikia S, Nasrabadi AM. Recognition of emotional states induced by music videos based on nonlinear feature extraction and som classification. In: 2014 21th Iranian Conference on Biomedical Engineering (ICBME). Tehran, Iran: IEEE; 2014:333-7. doi: 10.1109/ICBME.2014.7043946.

27. Yuvaraj R, Murugappan M, Ibrahim NM, Sundaraj K, Omar $\mathrm{Ml}$, Mohamad K, et al. Optimal set of EEG features for emotional state classification and trajectory visualization in Parkinson's disease. Int J Psychophysiol. 2014;94(3):482-95. doi: 10.1016/j.ijpsycho.2014.07.014.

28. Khalili Z, Moradi MH. Emotion recognition system using brain and peripheral signals: using correlation dimension to improve the results of EEG. In: Neural Networks, 2009. IJCNN 2009. International Joint Conference on 2009 Jun 14. Atlanta, GA, USA: IEEE; 2009:1571-5. doi: 10.1109/ IJCNN.2009.5178854.

29. Wang XW, Nie D, Lu BL. Emotional state classification from EEG data using machine learning approach. Neurocomputing. 
2014;129:94-106. doi: 10.1016/j.neucom.2013.06.046

30. Jie X, Cao R, Li L. Emotion recognition based on the sample entropy of EEG. Biomed Mater Eng. 2014;24(1):1185-92. doi: 10.3233/bme-130919.

31. Hosseinifard B, Moradi MH, Rostami R. Classifying depression patients and normal subjects using machine learning techniques and nonlinear features from EEG signal. Comput Methods Programs Biomed. 2013;109(3):339-45. doi: 10.1016/j.cmpb.2012.10.008.

32. Murugappan M, Nagarajan R, Yaacob S. Combining spatial filtering and wavelet transform for classifying human emotions using EEG Signals. J Med Biol Eng. 2011;31(1):45-51. doi: 10.5405/jmbe.710.

33. Sourina O, Liu Y. A Fractal-based Algorithm of Emotion Recognition from EEG using Arousal-Valence Model. In: Proceeding of the International Conference on Bio-Inspired Systems and Signal Processing. Rome, Italy: Biosignals; 2011:209-14

34. Chen DW, Han N, Chen JJ, Guo H. Novel algorithm for measuring the complexity of electroencephalographic signals in emotion recognition. J Med Imaging Health Inform. 2017;7(1):203-10. doi: 10.1166/jmihi.2017.2007.

35. Lan Z, Sourina O, Wang L, Liu Y. Real-time EEG-based emotion monitoring using stable features. Vis Comput. 2016;32(3):347-58. doi: 10.1007/s00371-015-1183-y.

36. Liu Y, Sourina O. EEG-based subject-dependent emotion recognition algorithm using fractal dimension. In: Systems, Man and Cybernetics (SMC), 2014 IEEE International Conference on 2014 Oct 5. San Diego, CA, USA: IEEE; 2014:3166-71. doi: 10.1109/SMC.2014.6974415.

37. Natarajan K, Acharya UR, Alias F, Tiboleng T, Puthusserypady SK. Nonlinear analysis of EEG signals at different mental states. Biomed Eng Online. 2004;3(1):7. doi: 10.1186/1475925x-3-7.

38. Maity AK, Pratihar R, Mitra A, Dey S, Agrawal V, Sanyal S, et al. Multifractal detrended fluctuation analysis of alpha and theta EEG rhythms with musical stimuli. Chaos Solitons Fractals. 2015;81:52-67. doi: 10.1016/j.chaos.2015.08.016.

39. Bahari F, Janghorbani A. Eeg-based emotion recognition using recurrence plot analysis and $\mathrm{k}$ nearest neighbor classifier. In: Biomedical Engineering (ICBME), 2013 20th Iranian Conference on 2013 Dec 18. Tehran, Iran: IEEE; 2013:22833. doi: 10.1109/ICBME.2013.6782224.

40. Zhuang N, Zeng Y, Tong L, Zhang C, Zhang H, Yan B. Emotion Recognition from EEG Signals Using Multidimensional Information in EMD Domain. Biomed Res Int. 2017;2017:8317357. doi: 10.1155/2017/8317357.

41. Li Y, Huang J, Zhou H, Zhong N. Human Emotion Recognition with Electroencephalographic Multidimensional Features by Hybrid Deep Neural Networks. Appl Sci. 2017;7(10):1060. doi: 10.3390/app7101060.

42. Ang $A Q$, Yeong $Y Q$, Wee W. Emotion Classification from EEG Signals Using Time-Frequency-DWT Features and ANN. J Comput Commun. 2017;5(3):75-9. doi: 10.4236/ jcc.2017.53009.

43. Mangala Gowri SG, Cyril Prasanna Raj P. Energy density feature extraction using different wavelets for emotion detection. Int J Appl Eng Res. 2018;13(1):520-7.

44. Eysenck SBG, Eysenck HJ, Barrett P. A revised version of the psychoticism scale. Pers Individ Dif. 1985;6(1):21-9. doi: 10.1016/0191-8869(85)90026-1.

45. Morris JD. Observations: SAM: the Self-Assessment Manikin; an efficient cross-cultural measurement of emotional response. J Advert Res. 1995;35(6):63-8.

46. Delorme A, Makeig S. EEGLAB: an open source toolbox for analysis of single-trial EEG dynamics including independent component analysis. J Neurosci Methods. 2004;134(1):9-21. doi: 10.1016/j.jneumeth.2003.10.009.

47. Sai CY, Mokhtar N, Arof $\mathrm{H}$, Cumming $\mathrm{P}$, Iwahashi $\mathrm{M}$. Automated Classification and Removal of EEG Artifacts With SVM and Wavelet-ICA. IEEE J Biomed Health Inform. 2018;22(3):664-70. doi: 10.1109/JBHI.2017.2723420.

48. Cao K, Guo Y, Su SW. A review of motion related EEG artifact removal techniques. In: Sensing Technology (ICST), 2015 9th International Conference on 2015 Dec 8. Auckland, New Zealand: IEEE; 2015:600-4.

49. Rahman FA, Othman MF, Shaharuddin NA. A review on the current state of artifact removal methods for electroencephalogram signals. In: Control Conference (ASCC), 2015 10th Asian 2015 May 31. Kota Kinabalu, Malaysia: IEEE; 2015:1-6.

50. Islam MK, Rastegarnia A, Yang Z. Methods for artifact detection and removal from scalp EEG: A review. Neurophysiol Clin. 2016;46(4-5):287-305. doi: 10.1016/j.neucli.2016.07.002.

51. Abdullah AK, Zhu ZC. Blind Source Separation Based of Brain Computer Interface System: A review. Res J Appl Sci Eng Technol. 2014;7(3):484-94.

52. Romo Vazquez R, Velez-Perez H, Ranta R, Louis Dorr V, Maquin D, Maillard L. Blind source separation, wavelet denoising and discriminant analysis for EEG artefacts and noise cancelling. Biomed Signal Process Control. 2012;7(4):389400. doi: 10.1016/j.bspc.2011.06.005.

53. Klemm M, Haueisen J, Ivanova G. Independent component analysis: comparison of algorithms for the investigation of surface electrical brain activity. Med Biol Eng Comput. 2009;47(4):413-23. doi: 10.1007/s11517-009-0452-1.

54. 54 Vorobyov S, Cichocki A. Blind noise reduction for multisensory signals using ICA and subspace filtering, with application to EEG analysis. Biol Cybern. 2002;86(4):293303. doi: 10.1007/s00422-001-0298-6.

55. Ng SC, Raveendran P. Enhanced Rhythm Extraction Using Blind Source Separation and Wavelet Transform IEEE Trans Biomed Eng. 2009;56(8):2024-34. doi: 10.1109/ TBME.2009.2021987.

56. Sweeney KT, McLoone SF, Ward TE. The use of ensemble empirical mode decomposition with canonical correlation analysis as a novel artifact removal technique. IEEE Trans Biomed Eng. 2013;60(1):97-105. doi: 10.1109/ tbme.2012.2225427.

57. Castellanos NP, Makarov VA. Recovering EEG brain signals: artifact suppression with wavelet enhanced independent component analysis. J Neurosci Methods. 2006;158(2):30012. doi: 10.1016/j.jneumeth.2006.05.033.

58. Hamaneh MB, Chitravas N, Kaiboriboon K, Lhatoo SD, Loparo KA. Automated removal of EKG artifact from EEG data using independent component analysis and continuous wavelet transformation. IEEE Trans Biomed Eng. 2014;61(6):1634-41. doi: 10.1109/tbme.2013.2295173.

59. Coifman RR, Donoho DL. Translation-invariant de-noising. In: Antoniadis A, Oppenheim G, eds. Wavelets and Statistics. New York, NY: Springer; 1995:125-50.

60. Akhtar MT, Mitsuhashi W, James CJ. Employing spatially constrained ICA and wavelet denoising, for automatic removal of artifacts from multichannel EEG data. Signal Processing. 2012;92(2):401-16. doi: 10.1016/j.sigpro.2011.08.005.

61. Kennel MB, Brown R, Abarbanel HD. Determining embedding dimension for phase-space reconstruction using a geometrical construction. Phys Rev A. 1992;45(6):3403-11.

62. Fraser AM, Swinney HL. Independent coordinates for strange attractors from mutual information. Phys Rev A Gen Phys. 1986;33(2):1134-40.

63. Atkinson J, Campos D. Improving BCl-based emotion 
recognition by combining EEG feature selection and kernel classifiers. Expert Syst Appl. 2016;47:35-41. doi: 10.1016/j. eswa.2015.10.049.

64. Shayegh F, Sadri S, Amirfattahi R, Ansari-Asl K. A model-based method for computation of correlation dimension, Lyapunov exponents and synchronization from depth-EEG signals. Comput Methods Programs Biomed. 2014;113(1):323-37. doi: 10.1016/j.cmpb.2013.08.014.

65. Naji M, Firoozabadi M, Azadfallah P. Classification of music-induced emotions based on information fusion of forehead biosignals and electrocardiogram. Cognit Comput. 2014;6(2):241-52. doi: 10.1007/s12559-013-9239-7.

66. Marwan N, Carmen Romano M, Thiel M, Kurths J. Recurrence plots for the analysis of complex systems. Phys Rep. 2007;438(5-6):237-329. doi: 10.1016/j.physrep.2006.11.001.

67. Zbilut JP, Webber CL. Embeddings and delays as derived from quantification of recurrence plots. Phys Lett A. 1992;171(34):199-203. doi: 10.1016/0375-9601(92)90426-M.

68. Webber CL, Jr., Zbilut JP. Dynamical assessment of physiological systems and states using recurrence plot strategies. J Appl Physiol (1985). 1994;76(2):965-73. doi: 10.1152/jappl.1994.76.2.965.

69. Guo R, Wang Y, Yan J, Yan H. Recurrence quantification analysis on pulse morphological changes in patients with coronary heart disease. J Tradit Chin Med. 2012;32(4):571-7.

70. Liu W, Zheng WL, Lu BL. Multimodal emotion recognition using multimodal deep learning. arXiv preprint arXiv:1602.08225. 2016.

71. Sharma R, Pachori R, Acharya U. Application of entropy measures on intrinsic mode functions for the automated identification of focal electroencephalogram signals. Entropy. 2015;17(2):669-91. doi: 10.3390/e17020669.

72. Pincus SM. Approximate entropy as a measure of system complexity. Proc Natl Acad Sci U S A. 1991;88(6):2297-301.

73. Peng CK, Havlin S, Stanley HE, Goldberger AL. Quantification of scaling exponents and crossover phenomena in nonstationary heartbeat time series. Chaos. 1995;5(1):82-7. doi: 10.1063/1.166141.

74. Zangeneh Soroush M, Maghooli K, Setarehdan SK, Motie Nasrabadi A. A novel method of eeg-based emotion recognition using nonlinear features variability and Dempster-Shafer theory. Biomed Eng Appl Basis Commun. 2018;30(4):1850026. doi: 10.4015/S1016237218500266.

75. Yang J, Honavar V. Feature subset selection using a genetic algorithm IEEE Intell Syst. 1998;13(2):44-9. doi: 10.1109/5254.671091.

76. Shi Y, Eberhart R. A modified particle swarm optimizer. In: Evolutionary Computation Proceedings, 1998. IEEE World Congress on Computational Intelligence., The 1998 IEEE International Conference on 1998 May 4. Anchorage, AK, USA: IEEE; 1998:69-73.

77. Lim CP, Jain LC. Advances in swarm intelligence. In: Lim CP, Jain LC, Dehuri $S$, eds. Innovations in Swarm Intelligence. Berlin, Heidelberg: Springer; 2009:1-7.

78. Lee K, Joo J, Yang J, Honavar V. Experimental comparison of feature subset selection using GA and ACO algorithm. In: International Conference on Advanced Data Mining and Applications 2006 Aug 14. Berlin, Heidelberg: Springer; 2006:465-72.

79. Morvant E, Habrard A, Ayache S. Majority vote of diverse classifiers for late fusion. In: Joint IAPR International Workshops on Statistical Techniques in Pattern Recognition (SPR) and Structural and Syntactic Pattern Recognition (SSPR) 2014 Aug 20. Berlin, Heidelberg: Springer; 2014:153-62.

80. Koelstra S, Muhl C, Soleymani M, Lee JS, Yazdani A, Ebrahimi $\mathrm{T}$, et al. Deap: A database for emotion analysis; using physiological signals. IEEE Trans Affect Comput. 2012;3(1):1831. doi: 10.1109/T-AFFC.2011.15. 